\title{
Mechanisms of Dexmedetomidine in Neuropathic Pain
}

\author{
Yang Zhao*, Jianshuai He, Ning Yu, Changxin Jia and Shilei Wang* \\ Department of Anesthesiology, The Affiliated Hospital of Qingdao University, Qingdao, China
}

\section{OPEN ACCESS}

Edited by:

Nick Andrews,

Harvard Medical School

United States

Reviewed by:

Dara Bree,

Cygnal Therapeutics, United States

Alan Kaye,

Louisiana State University Health

Sciences Center Shreveport,

United States

*Correspondence:

Yang Zhao

zhaoy1979@163.com

Shilei Wang

wshlei@aliyun.com

Specialty section: This article was submitted to

Neuropharmacology,

a section of the journal

Frontiers in Neuroscience

Received: 10 December 2019

Accepted: 20 March 2020

Published: 05 May 2020

Citation:

Zhao Y, He J, Yu N, Jia C and

Wang S (2020) Mechanisms

of Dexmedetomidine in Neuropathic

Pain. Front. Neurosci. 14:330.

doi: 10.3389/fnins.2020.00330
Dexmedetomidin is a new-generation, highly selective $\alpha 2$ adrenergic receptor agonist with a large number of advantages, including its sedative and analgesic properties, its ability to inhibit sympathetic nerves, its reduced anesthetic dosage, its hemodynamic stability, its mild respiratory depression abilities, and its ability to improve postoperative recognition. Its safety and effectiveness, as well as its ability to provide a certain degree of comfort to patients, make it a useful anesthetic adjuvant for a wide range of clinical applications. For example, dexmedetomidine is commonly used in patients undergoing general anesthesia, and it also exerts sedative effects during tracheal intubation or mechanical ventilation in intensive care unit patients. In recent years, with the deepening of clinical research on dexmedetomidine, the drug is still applied in the treatment of spastic pain, myofascial pain, neuropathic pain, complex pain syndrome, and chronic headache, as well as for multimodal analgesia. However, we must note that the appropriateness of patient and dose selection should be given attention when using this drug; furthermore, patients should be observed for adverse reactions such as hypotension and bradycardia. Therefore, the safety and effectiveness of this drug for long-term use remain to be studied. In addition, basic experimental studies have also found that dexmedetomidine can protect important organs, such as the brain, heart, kidney, liver, and lung, through various mechanisms, such as antisympathetic effects, the inhibition of apoptosis and oxidative stress, and a reduction in the inflammatory response. Moreover, the neuroprotective properties of dexmedetomidine have received the most attention from scholars. Hence, in this review, we mainly focus on the characteristics and clinical applications of dexmedetomidine, especially the role of dexmedetomidine in the nervous system and the use of dexmedetomidine in the relief of neuropathic pain.

Keywords: neuropathic pain, dexmedetomidine, adrenergic receptor, neuroprotective effect, $\alpha 2$ adrenergic receptor

\section{DEXMEDETOMIDINE}

\section{Discovery and Clinical Application of Dexmedetomidine}

Dexmedetomidine is a dextrorotatory isomer of the racemic mixture medetomidine (Keating, 2015). Hoy et al. investigated whether the activation of $\alpha 2$-adrenergic receptors could change the amount of inhaled anesthetic, found that the $\alpha 2$-adrenergic receptor agonist dexmedetomidine itself was an anesthetic with a calming and hypnotic effect (Hoy and Keating, 2011). Then, they immediately recruited relevant patents and further launched a series of studies, 
and the relevant research results were also utilized to the human body, which led to the emergence of dexmedetomidine as a sedative (Barends et al., 2017). It was subsequently developed in 1990 by OrionPharma (Finland) and Abbott Labs (United States), and afterward, it was used in intensive care units (ICUs) as a short-term sedative $(<24 \mathrm{~h})$ approved by the United States government in 1999 (Keating, 2015). In 2008, dexmedetomidine was approved for non-tracheal intubation patients and perioperative treatment (Cruickshank et al., 2016). However, in recent years, accumulating evidence has demonstrated that, clinically, dexmedetomidine has an analgesic effect on ischemic pain, acute postoperative pain, refractory cancer pain, and herpetic stomatitis after living lung transplantation (Hoy and Keating, 2011). In addition, a large number of studies in different animal models have also confirmed that dexmedetomidine has analgesic and antiinflammatory effects in postoperative, streptozotocin-induced, Freund's complete adjuvant, formalin, and carrageenan-induced inflammation as well as acute nociceptive and neuropathic pain (Mahmoud and Mason, 2015; Sottas and Anderson, 2017).

\section{Basic Pharmacology of Dexmedetomidine}

\section{Structure of Dexmedetomidine}

Dexmedetomidine is a newly developed $\alpha 2$ adrenergic receptor agonist, an imidazole derivative with the chemical name 4-[(1R)1-(2,3-dimethylphenyl)ethyl]-3H-imidazole hydrochloride (Greenberg et al., 2017). Dexmedetomidine is a highly potent and highly selective $\alpha 2$ adrenergic receptor agonist with a receptor selectivity $(\alpha 2 / \alpha 1)$ of $1,620: 1$, but clonidine receptor selectivity $(\alpha 2 / \alpha 1)$ is 220:1 (Giovannitti et al., 2015). Moreover, the intrinsic activity of dexmedetomidine is stronger than that of clonidine (3:1) (Pichot et al., 2012). The elimination half-life of dexmedetomidine is approximately $2 \mathrm{~h}$, while the elimination half-life of clonidine is $8.6 \pm 1.5 \mathrm{~h}$. Thus, due to its unique receptor activity and short half-life, as well as its special pharmacological effects, dexmedetomidine has broad application prospects in clinical anesthesia (Mavropoulos et al., 2014; Greenberg et al., 2017).

\section{Specificity of the Dexmedetomidine Effect: Acceptor-Mediated Entrance Into the Neuronal Cytosol}

Dexmedetomidine is a G-protein-coupled $\alpha 2$ receptor agonist (Rodriguez-Gonzalez et al., 2016). The $\alpha 2$ adrenergic receptors in humans mainly contain $\alpha 2 \mathrm{~A}, \alpha 2 \mathrm{~B}$, and $\alpha 2 \mathrm{C}$ and are widely distributed in the central nervous system (CNS), peripheral nervous system (PNS), autonomic ganglia, and other organ tissues, including the blood vessels, liver, kidney, pancreas, and platelets (Baron, 2009). Different subtypes of $\alpha 2$ adrenergic receptors have different functions. The $\alpha 2 \mathrm{~A}$ receptor is considered to be the major presynaptic inhibitory feedback receptor, controlling the exocytosis of adrenergic neurons (Scholz et al., 2019). Thus, deletion of a gene encoding the $\alpha 2 \mathrm{~A}$ receptor results in elevated blood pressure, increased heart rate, and easy progression to cardiac hypertrophy and heart failure. In addition, the $\alpha 2 \mathrm{~A}$ receptor or the $\alpha 2$ receptor agonist is required for sedation, analgesia, epilepsy regulation, and platelet aggregation (Baron, 2009; Scholz et al., 2019). The $\alpha 2 \mathrm{~B}$ receptor is mainly distributed in peripheral vascular smooth muscle and can cause a transient hypertensive response after activation (Jiang et al., 2017). The $\alpha 2 \mathrm{~B}$ receptor in the spinal cord is a basic component of the regulation of nitrogen monoxide analgesia by descending noradrenergic neurons (Jiang et al., 2017; Mantz et al., 2011). The $\alpha 2 \mathrm{C}$ receptor is mainly distributed in the hippocampus, basal ganglia, olfactory bulb system, and cerebral cortex and is involved in the regulation of a variety of complex memory and behavioral functions (Mantz et al., 2011). Dexmedetomidine is a highly selective activator of the $\alpha 2 \mathrm{~A}$ receptor, acting on the nucleolus of the nucleus, and it can have a sedative and hypnotic effect; its action on the spinal cord can produce analgesic effects, while its action on the peripheral and CNSs can play a role in inhibiting sympathetic excitation (Kenney et al., 2014; Giovannitti et al., 2015).

\section{Inhibition of Neurotransmitter Release}

In the CNS, the most important method of synaptic transmission is neurochemical transmission (Pocock and Kettenmann, 2007). The neurotransmitter, in synaptic transmission, is a specific chemical that acts as a "messenger" (Lee, 2013). It is bound to the corresponding postsynaptic membrane receptor immediately after release from the presynaptic membrane, producing synaptic depolarization potentials or superpolar potentials, resulting in increased or decreased excitability of the postsynaptic nerve (Zindler and Zipp, 2010; Lee, 2013). Thus, the release of neurotransmitters is directly related to the production of neural excitability. Previous studies have shown that the release of neurotransmitters, such as catecholamine and norepinephrine, is closely associated with hypoxic brain damage (Zindler and Zipp, 2010). Catecholamine increases neuronal sensitivity to glutamate during neuronal ischemia, while norepinephrine metabolites promote oxidative stress, which further exacerbates damage to nerve tissue (Starke, 2014). Furthermore, it was discovered that dexmedetomidine could reduce the release of catecholamines in nerve endings by reducing the activity of the sympathetic nervous system (Wu et al., 2020). It was also found to directly act on $\alpha 2$ receptors in monoaminergic neurons and dendrites in the brain to inhibit the secretion of catecholamines (Obata, 2017). Hence, the above study concluded that dexmedetomidine could reduce neuronal damage by inhibiting neurotransmitter release, which improved ischemic perfusion and metabolic disorders (Wang et al., 2019).

\section{Modulation of Voltage-Gated Calcium Channels and Glutamate Excitotoxicity}

Excessive release of glutamate may induce an increase in intracellular $\mathrm{Ca}^{2+}$ expression levels, resulting in increased production of oxygen free radicals, thereby interfering with mitochondrial function and activating proteases, ultimately leading to neuronal death (Crupi et al., 2019). Wang et al. (2019) found that dexmedetomidine could upregulate the expression of excitatory amino acid transporter 1 by increasing the release of the $N$-methyl-D-aspartate receptor and then that it could trigger 
a decrease in glutamate release to alleviate nerve cell damage in neonatal rats caused by isoflurane. Therefore, this study implies that dexmedetomidine might exert a neuroprotective effect by inhibiting the influx of calcium ions and promoting the release of glutamate. Furthermore, another study reported that dexmedetomidine could enhance the expression of excitatory amino acid transporter 3 (EAAT3) in glutamate in Xenopus oocytes in a concentration-dependent manner, suggesting that dexmedetomidine could decrease the concentration of extracellular glutamate by increasing the expression of EAAT3 and could exert neuroprotective and anticonvulsant effects (Do et al., 2014). These effects were mediated by protein kinase $\mathrm{C}$ and phosphatidylinositol-3-hydroxykinase. Additionally, KM Chiu et al. (2011) observed that dexmedetomidine activated $\alpha 2 \mathrm{~A}$ adrenergic receptors in cerebral cortical nerve endings, inhibited glutamate release, and further reduced extracellular glutamate deposition by suppressing the activity of N-type and P/Q-type voltage-gated calcium channels and mitogenactivated protein kinases (MAPKs), which ultimately produced neuroprotective effects.

\section{NEUROPATHIC PAIN}

Neuropathic pain, a type of chronic and potentially disabling pain caused by disease or injury to the somatosensory nervous system and spinal cord, can be accompanied by various chronic conditions, such as viral infections (e.g., postherpetic neuralgia), autoimmune diseases, cancers, and metabolic disorders (e.g., diabetes mellitus); neuropathic pain is one of the most intense types of chronic pain, resulting in a major economic burden and a serious public health issue, with an estimated prevalence of 7$10 \%$ adults worldwide (Cohen and Mao, 2014; Knezevic et al., 2017). According to the underlying etiology and the anatomical location of the specific lesion, clinical neuropathic pain is divided into four main types, namely, painful peripheral neuropathies, central pain syndromes, complex painful neuropathic disorders, and mixed-pain syndromes (Baron, 2009; Binder and Baron, 2016). Painful peripheral neuropathies are caused by ischemic, traumatic, inflammatory, infectious, metabolic, or compressive damage to the PNS, such as polyneuritis, phantom limb pain, diabetic peripheral neuralgia, and postherpetic neuralgia (Stacey, 2005; Brouwer et al., 2014; Scholz et al., 2019). Central pain syndromes are characterized by primary pain resulting from dysfunction or damage to the CNS pain conduction pathway, mainly involving cerebrovascular disease, spinal cord trauma, etc. (Bettini and Moore, 2016; Scholz et al., 2019). Complex painful neuropathic disorders are also called "complex regional pain syndromes (CRPS)," which may develop as a disproportionate consequence of trauma typically affecting the limbs (Colloca et al., 2017). Mixed pain syndromes are characterized by a combination of nociceptive and neuropathic pain (Baron, 2009). Currently, the incidence of neuropathic pain is high, and treatment is difficult, which seriously affects the work and quality of life of patients (Vranken, 2012; Yan et al., 2017). Nevertheless, the pathogenesis of neuropathic pain is complex and has not yet been clarified.

\section{NEUROPROTECTIVE EFFECTS AND MECHANISMS OF DEXMEDETOMIDINE}

\section{Neuroprotective Effect of Dexmedetomidine}

In recent years, dexmedetomidine has received extensive attention from scholars due to its good sedative effect (Keating, 2015). Moreover, it has been widely used as an anesthesia adjuvant for clinical anesthesia and severe disease (Baron, 2009). Additionally, growing evidence has also shown that dexmedetomidine can produce neuroprotective effects through various mechanisms, such as antioxidation and anti-infection activities, the inhibition of apoptosis, the promotion of neurogenesis, and the influence of cell signaling pathways (Shen et al., 2017; Wu et al., 2018). The neuroprotective effect of dexmedetomidine was first proposed by Hoffman et al. (1993). It was found that, in a rat model of cerebral ischemia, intraperitoneal injection of dexmedetomidine before cerebral ischemia significantly reduced plasma catecholamine levels, thereby reducing cerebral ischemic damage (Rodriguez-Gonzalez et al., 2016; Wang S.L. et al., 2017). Subsequently, the neuroprotective effect of dexmedetomidine was further examined by a large number of researchers (Rodriguez-Gonzalez et al., 2016; Jiang et al., 2017). For example, Sato et al. (2010) uncovered that the neurological function of ischemic injury was significantly improved in rats with focal cerebral ischemia after dexmedetomidine, the survival of neurons in the CA1 region of the ischemic hippocampus increased, and dexmedetomidine combined with hypothermia could enhance the above effects. Zhu et al. (2013) revealed that dexmedetomidine was applied in a rat model of cerebral ischemia-reperfusion injury, which significantly reduced neurological deficits, cerebral infarction volume, cerebral edema, and neuronal death in the hippocampal CA1 and cerebral cortex. Liao et al. (2014) discovered that dexmedetomidine could decrease isoflurane-induced neuroapoptosis by activating the phosphatidylinositol 3-kinase (PI3K)/protein kinase B (Akt) pathway and suppressing the p38/ MAPK pathway in the hippocampus of neonatal rats. Furthermore, the role of dexmedetomidine in neuropathic pain has also gradually been disclosed (Di Cesare Mannelli et al., 2017). For instance, intrathecal injection of dexmedetomidine relieved neuropathic pain through the modulation of MPK3/extracellular regulated protein kinase (ERK)1/2 in a mouse model of neuropathic pain (Qian et al., 2019), dexmedetomidine ameliorated neuropathic pain by hindering spinal P2X7R expression and ERK phosphorylation in an animal model of chronic constriction injury (Lin et al., 2018), and dexmedetomidine attenuated neuropathic hypersensitivity and inflammation partially by restraining $\mathrm{N}$-methyl-D-aspartate receptor 2B (NR2B), nuclear factor-kappa B (NF-кB), and inducible nitric oxide synthase (iNOS) expression in the spinal cord of rats with neuropathic pain resulting from chronic constriction injury of the sciatic nerve (Liang et al., 2017). Therefore, the neuroprotective effect of dexmedetomidine is unquestionable, 
but its specific neuroprotective mechanism has not yet been fully elucidated.

\section{Mechanism of Dexmedetomidine's Protective Effects on the Nervous System}

\section{Anti-inflammatory Influences of Dexmedetomidine}

Brain injury can cause the release of a variety of inflammatory factors, and the inflammatory response is an important pathogenesis of brain injury (Jassam et al., 2017; Magalhaes et al., 2019). The neuroprotective effect of dexmedetomidine is also related to its inhibition of the inflammatory response (Zhang et al., 2015). The immune response plays an important role in neuroprotection ( $\mathrm{Li}$ et al., 2017). Dillon et al. (2017) found that dexmedetomidine could reduce the levels of inflammatory cytokines, such as tumor necrosis factor- $\alpha$ (TNF- $\alpha$ ) and interleukin-1 (IL-1), by activating the $\alpha 2$ adrenergic receptor signaling pathway. Studies have shown that dexmedetomidine can attenuate the inflammatory responses associated with ischemia/reperfusion injury by inhibiting NF- $\kappa \mathrm{B}$ activation (Wang L. et al., 2017). In addition, Kim et al. (2017) discovered that dexmedetomidine could induce an antiinflammatory response in cerebral ischemia-reperfusion injury through inactivation of the Toll-like receptor 4 (TLR4)/NF- $\mathrm{KB}$ pathway, which is an important mechanism of neuroprotection.

\section{Anti-excitotoxicity Actions of Dexmedetomidine}

Cerebral ischemia and hypoxia can lead to increased release of glutamate and aspartate (Hsieh et al., 2017). If the concentration of glutamic acid and aspartic acid in the extracellular fluid is increasing, an excessive concentration of the excitatory amino acid neurotransmitter glutamate causes $\mathrm{N}$-methyl-D-aspartate receptor overactivation, which finally leads to neuronal cell apoptosis (Zhang et al., 2017). However, in the case of cerebral ischemia and hypoxia, dexmedetomidine could reduce the release of glutamate by acting on cell membrane ion channels and, on the other hand, could increase the production of brain-derived growth factor (BDNF) in astrocytes to protect against glutamate neurotoxicity (Liu et al., 2017). In addition, it was found that dexmedetomidine could not only reduce glutamate content after cerebral ischemia but also increase gamma aminobutyric acid content and decrease brain ultrastructural damage, indicating that dexmedetomidine might exert its neuroprotective effects by downregulating excitatory neurotransmitters and therefore upregulating inhibitory neurotransmitters (Alam et al., 2017; Endesfelder et al., 2017).

\section{Inhibition of Neuronal Apoptosis by Dexmedetomidine}

Neuronal necrosis often occurs early in ischemia and hypoxia damage, while neuronal apoptosis can continue to occur within a few weeks after ischemia (Sun et al., 2015; Li C. et al., 2018). Apoptosis is defined as programmed cell death mediated by many genes, among which the important apoptosis regulatory proteins are Bcl-2, BCL-XL, and Bax (Wang et al., 2020). Experimental studies have revealed that dexmedetomidine can increase BDNF expression by directly acting on the $\alpha 2 \mathrm{~A}$ receptor and then modulating the ERK1/2 pathway, and BDNF can in turn promote the expression of Bcl-2 and BCL-XL by activating the PI3K/Akt signaling pathway and MAPK pathway and thus suppressing the p38 and c-Jun N-terminal kinase (JNK) pathways, which eventually leads to a decrease in neuronal apoptosis (Bell et al., 2014; Li Z.C. et al., 2018). Moreover, Kuang et al. (2015) revealed that dexmedetomidine could effectively alleviate ketamine-induced neuronal apoptosis in the hippocampal CA1 region as well as learning and memory dysfunction in rat brain development, and dexmedetomidine alone had no obvious neurotoxicity in 7-day-old neonatal rats.

\section{Roles of Dexmedetomidine in Astroglia}

Glial cells are supportive cells widely distributed in the nervous system (Watzlawik et al., 2010). They support and guide neuronal migration, participate in the repair and regeneration of the nervous system or immune responses, form myelin and bloodbrain barriers, and maintain potassium ion concentrations outside nerve cells (Watzlawik et al., 2010; Machado et al., 2016). Glial cells include astrocytes, oligodendrocytes, and microglia in the CNS (von Bernhardi et al., 2016). In the CNS, astrocytes connect capillaries and neurons through perivascular limbs, which can transport nutrients and eliminate metabolites to neurons (Zuchero and Barres, 2015). It has been found that dexmedetomidine can inhibit the astrocyte-induced overactivation caused by ischemia-reperfusion and can reduce the expression of glial fibrillary acidic protein and inflammatory factor TNF- $\alpha$, which suggests that dexmedetomidine has neuroprotective effects on cerebral ischemia-reperfusion injury in rats (Freeman et al., 2015; Shi et al., 2018).

\section{Antioxidative Effects of Dexmedetomidine}

The brain is the most oxygen consuming of all organs in the human body but lacks antagonists against oxidative stress in CNS neurons, so neuronal cells are easily damaged by the release of free radicals (Salim, 2017; Dumitrescu et al., 2018). Cerebral ischemia and hypoxia damage can result in an increase in reactive oxygen species in the brain tissue, which in turn causes oxidative damage to cellular macromolecules such as nucleic acids and proteins to induce cell death (TorresCuevas et al., 2019; Zhou et al., 2019). Studies have found that inhalation of high-concentration oxygen in rat pups produces inflammatory brain damage induced by oxidative stress, which is characterized by increased apoptosis, a decreased ratio of glutathione/oxidized glutathione disulfide, increased lipid peroxidation, and increased IL-1 $\beta$ levels (Zhenyukh et al., 2017). Nevertheless, dexmedetomidine treatment has been observed to alleviate or eliminate these deleterious effects induced by these oxidation reactions (Fu et al., 2017; Xu et al., 2019).

\section{Dexmedetomidine Regulates Synaptic Plasticity and Hippocampal Long-Term Potentiation}

Synaptic plasticity, including structural and functional plasticity, provides an important basis for learning and memory (Singh and Abraham, 2017). However, hippocampal long-term potentiation refers to the long-term enhancement of synaptic transmission efficiency produced by the tonic stimulation of neural pathways, 
which can reflect the physiological activities of neurons during the formation of learning and memory and is a manifestation of synaptic plasticity (MacDonald et al., 2006). Previous research reported that 7 -day-old rats were given moderate doses of dexmedetomidine, causing mild respiratory depression, but these doses did not impair synaptic plasticity in adulthood, which implied that dexmedetomidine might be relatively safe during development (Tachibana et al., 2012). Additionally, cerebral ischemia and hypoxia could cause longterm potentiation after ischemia (i-LTP), while the combined application of dexmedetomidine could not only restrain the release of noradrenaline and glutamate from the presynaptic membrane, which triggered presynaptic inhibition and weakened i-LTP, but also hyperpolarize the postsynaptic membrane by reducing the activation of the $N$-methyl-D-aspartate receptor and hindering the $\beta$-receptor and its downstream cyclic AMP (cAMP)/protein kinase A (PKA) pathway; thus, $\beta$-cAMP/PKA intracellular signaling pathways might be involved in the regulation of improving synaptic plasticity in response to dexmedetomidine (Niittykoski et al., 2000; Takamatsu et al., 2008; Kato et al., 2014).

\section{Suppression of Sympathetic Activity and Regulation of Cerebral Blood Flow}

Studies have shown that dexmedetomidine reduces cerebral blood flow and the brain metabolic rate in healthy volunteers (Wang et al., 2013). In patients who undergo surgery, dexmedetomidine can lessen intracranial pressure, reduce cerebral oxygen metabolism, maintain the balance of cerebral oxygen supply and demand, and diminish agitation and postoperative sputum (Jiang et al., 2017). Cheng et al. also confirmed that in cerebral ischemia-reperfusion, dexmedetomidine decreased the area of cerebral infarction in the early stage of ischemia-reperfusion by reducing the heterogeneity of mixed venous oxygen saturation, reducing regional cerebral blood flow and cerebral oxygen consumption, and maintaining cerebral oxygen supply and demand (Cheng et al., 2018). In addition, it has been reported that dexmedetomidine is dependent on the $\alpha 2$ adrenergic receptor and preferentially bound to the inactivated sodium channel and acetylcholine receptor channel in the supraspinatus ganglion in rats and that it then inhibits sodium channels and acetylcholine receptor channels in a dose-dependent manner (Yang et al., 2015). Moreover, it has been further demonstrated that the inhibition of dexmedetomidine in sodium channels and acetylcholine receptor channels cannot be blocked by yohimbine, phentolamine, or atropine, which might be suggestive of the mechanism of the central antisympathetic activity of dexmedetomidine (Yang et al., 2015).

\section{Induction of the Low-Temperature Effect of Dexmedetomidine}

McAdams et al. (2015) administered dexmedetomidine or combined hypothermia treatment to neonatal rats and subsequently discovered that acute or persistent exposure to dexmedetomidine did not cause abnormal pathological changes in the brain tissue of newborn rats, and there was a correlation between plasma dexmedetomidine concentration and brain tissue dexmedetomidine concentration. Furthermore, it was also found that the dexmedetomidine level in rat brain tissue reached a peak within 15 min of administration in the low-temperature group, and the central temperature was reduced from 32 to $30^{\circ} \mathrm{C}$ within $30 \mathrm{~min}$ after administration, indicating that dexmedetomidine combined with hypothermia might be used to treat acute craniocerebral injury (Lewis et al., 2015).

\section{AN OVERVIEW OF THE CLINICAL EVIDENCE OF DEXMEDETOMIDINE ANALGESIC EFFICACY}

\section{Molecular Mechanism of Analgesic Efficacy of Dexmedetomidine}

There are four different types of $\alpha 2$ adrenergic receptor subtypes in the human body, namely, $\alpha 2 \mathrm{~A}, \alpha 2 \mathrm{~B}, \alpha 2 \mathrm{C}$, and $\alpha 2 \mathrm{D}$ receptors (Giovannitti et al., 2015). Current studies suggest that the $\alpha 2 \mathrm{~A}$ receptor mediates the anesthetic and analgesic effects of dexmedetomidine (Mantz et al., 2011). The mechanism of dexmedetomidine analgesia has not been fully clarified, but the main mechanisms might be as follows: (1) peripheral analgesic effect, an analgesic effect produced by inhibiting the transmission of pain signals by inhibiting $A \delta$ and $C$ fibers; (2) central analgesic effect, mainly depolarizes the blue plaque and the descending noradrenergic pathway of the spinal cord to the presynaptic membrane, inhibiting the release of substance $\mathrm{P}$ and other nociceptive peptides in the presynaptic membrane and thereby inhibiting the spinal cord via the transmission of angular noxious stimuli, which in turn terminates the signaling of pain; and (3) local analgesic effect, modulation of hyperalgesia by stimulating the $\alpha 2$ receptor (Zhang and Bai, 2014). In the past, opioids were mainly used to relieve acute postoperative pain, but opioids can cause toxic side effects such as nausea, vomiting, respiratory depression, and itchy skin (Gupta and Bah, 2016). However, the highly selective $\alpha 2$ receptor agonist dexmedetomidine has good sedative and analgesic effects, has little effect on the circulatory system, and does not inhibit breathing (Giovannitti et al., 2015). It is now being used as an auxiliary drug due to its good anesthesia and analgesia (Cohen and Mao, 2014). In terms of postoperative multimodal analgesia, dexmedetomidine reduces the amount of opioids used, and patient comfort is high (Hoy and Keating, 2011; Cohen and Mao, 2014). Although the current literature reports that dexmedetomidine only plays a supporting role in analgesia, the mode of administration of dexmedetomidine plus other analgesics has brought new ideas and compatibility to postoperative analgesia and promotes the improvement of pain treatment (Vranken, 2012). The advantages of dexmedetomidine for postoperative analgesia primarily include the following: (1) the diversity of analgesic methods, dexmedetomidine can be administered not only by intravenous or intrathecal administration but also by topical medication, which can enhance the postoperative analgesic effect; (2) it is the only sedative that has an analgesic effect and has 
a wide range of safety, dexmedetomidine not only enhances postoperative analgesia but also prevents postoperative sedation; and (3) the wide range of applications and organ protection, dexmedetomidine can be used for postoperative analgesia in various operations. It can be combined with analgesics with different mechanisms of action to exert its superior effects, such as reducing the incidence of postoperative agitation and spasm (Pichot et al., 2012). However, in addition to the above advantages, dexmedetomidine has side effects in its range of conventional use. Among them, the most common side effects are bradycardia and hypotension (Weerink et al., 2017). Therefore, the dosage and the mode of administration should be strictly controlled. If a high concentration of the load is given, hypotension, high blood pressure, or bradycardia can result.

At present, in view of the better analgesic effect of dexmedetomidine, in addition to being used for surgical analgesia, the drug can also be used for labor analgesia, gastrointestinal endoscopy analgesia, neuropathic pain, cancer pain, and so on (Hoy and Keating, 2011; Vranken, 2012). Moreover, a growing number of studies have confirmed that dexmedetomidine has an analgesic effect on neuropathic pain, and its mechanism may be related to the downregulation of the microglia $\mathrm{P} 2 \mathrm{X} 4$ receptor, phosphorylated p38 protein kinase, and brain-derived neurotrophic factor (Zhou et al., 2014). The detailed studies of dexmedetomidine in relation to neuropathic pain are listed in Table $\mathbf{1 .}$

\section{Clinical Evidence of Dexmedetomidine Analgesic Efficacy}

Moreover, there have been some randomized clinical trials on the use of dexmedetomidine for neuropathic pain, which are exhibited in Table 2. Andjelkovic et al. conducted a randomized controlled clinical trial to evaluate the analgesic efficacy of

TABLE 1 | Studies of dexmedetomidine for neuropathic pain.

Model
A mouse model induced by chronic
constriction injury
A randomized prospective
double-blinded trial
Rats diabetic neuropathy pain mode

A rat model induced by chronic constriction injury

A rat model induced by chronic constriction injury

A rat model induced by chronic constriction injury

A rat model of skin/muscle incision and retraction

A rat model of neuropathic pain

Rats with nerve-ligation injury

A patient with metastatic sarcoma suffering from an acute postoperative neuropathic pain crisis

Rats with spared nerve injury

Rats subjected to chronic constriction injury

A rat spinal nerve ligation model

Vincristine-evoked painful neuropathic rats

A rat model of neuropathic pain induced by spinal nerve ligation

Nerve injury-induced pain

The spinal nerve ligation model of neuropathic pain in rats

\section{Results}

Dexmedetomidine play an analgesic role in neuropathic pain through the regulation of MKP3/ERK1/2 signaling pathway.

Dexmedetomidine is considered as an adjuvant to bupivacaine paravertebral block and further reliver pain during the early postoperative hours, and it provided a better effect on chronic postoperative pain.

Dexmedetomidine alleviated diabetic neuropathy pain in rats via hindering inflammation and astrocyte activation, which may be attributed to downregulation of the Wnt $10 \mathrm{a} / \beta$-catenin signaling pathway.

The intrathecal injection of dexmedetomidine may improve the behavioral ability of rats with chronic neuralgia and reduce the degree of pain, which may be associated with the inhibition of the expression of PKC in the spinal dorsal horn. Dexmedetomidine changed the expressions of NR2B and GABAA- $\alpha 1$ following peripheral nerve injury in rats to manage neuropathic pain.

Dexmedetomidine relieves neuropathic pain by inhibiting

hyperpolarization-activated cyclic nucleotide-gated currents in dorsal root ganglia neurons.

Peripheral administration of dexmedetomidine improves mechanical and heat hyperalgesia and mitigates postoperative pain.

The therapeutic effectiveness of dexmedetomidine in neuropathic pain may be through inhibition of proinflammatory cytokines, primarily IL- 6 and TNF- $\alpha$.

The antinociceptive of dexmedetomidine was confirmed in neuropathic pain treatment.

Dexmedetomidine should be considered as a viable treatment alternative for acute postoperative neuropathic pain crisis.

Dexmedetomidine could attenuate the neuropathic pain via down-expressing P2X4Rs, p-p38, and BDNF in microglia of spinal dorsal horn.

Dexmedetomidine played an anti-nociceptive effect via mediating IL-18 signaling pathway in microglia.

Intraplantar injection of dexmedetomidine produced an antiallodynic effect in spinal nerve ligation-induced neuropathic pain.

Dexmedetomidine shows a dose-dependent antiallodynic effect on mechanical and cold stimuli in vincristine-evoked neuropathic rat models.

Dexmedetomidine decreases hyperalgesia in neuropathic pain by increasing acetylcholine in the spinal cord.

Dexmedetomidine exerts antinociceptive effects in the development of neuropathic pain after peripheral nerve injury.

Systemic administration with dexmedetomidine could prevent and treat the nerve injury-induced pain.

\section{References}

Qian et al., 2019

Abd-Elshafy et al., 2019

Zhong et al., 2018

Li and Zhang, 2018

Chen et al., 2018

Yang et al., 2018

Huang et al., 2017

Farghaly et al., 2016

Farghaly et al., 2014

O'Neil et al., 2014

Zhou et al., 2014

Chandrasekar et al., 2005

Lee et al., 2013

Park et al., 2012

Kimura et al., 2012

Ulger et al., 2009

Ma et al., 2018 
TABLE 2 | Randomized clinical trials of dexmedetomidine for neuropathic pain.

\begin{tabular}{|c|c|c|c|}
\hline Pain condition & $\begin{array}{l}\text { Number of participants and } \\
\text { does/delivery of route }\end{array}$ & Primary outcomes & References \\
\hline $\begin{array}{l}\text { Perioperative opioid } \\
\text { consumption in laparoscopic } \\
\text { intestine resection }\end{array}$ & $\begin{array}{l}59 \text { participants, continuous intravenous } \\
\text { infusion of dexmedetomidine } \\
(0.5 \mu \mathrm{g} / \mathrm{kg} / \mathrm{h})\end{array}$ & $\begin{array}{l}\text { Dexmedetomidine reduced intraoperative propofol } \\
\text { consumption. }\end{array}$ & $\begin{array}{l}\text { Andjelkovic et al., } \\
2018\end{array}$ \\
\hline $\begin{array}{l}\text { Postoperative pain and bowel } \\
\text { function recovery after } \\
\text { abdominal hysterectomy }\end{array}$ & $\begin{array}{l}240 \text { women, Received } \\
\text { dexmedetomidine infusion }(0.5 \mu \mathrm{g} / \mathrm{kg} \\
\text { loading, } 0.4 \mu \mathrm{g} / \mathrm{kg} / \mathrm{h} \text { infusion) }\end{array}$ & $\begin{array}{l}\text { Dexmedetomidine infusion improved postoperative pain } \\
\text { and enhanced recovery of bowel function undergoing } \\
\text { abdominal hysterectomy. }\end{array}$ & Xu et al., 2017 \\
\hline $\begin{array}{l}\text { Postoperative opioid } \\
\text { consumption and pain after } \\
\text { major spine surgery }\end{array}$ & $\begin{array}{l}142 \text { participants, } 1 \mu \mathrm{g} / \mathrm{kg} \text { load followed } \\
\text { by a continuous infusion of } 0.5 \mu \mathrm{g} / \mathrm{kg} / \mathrm{h}\end{array}$ & $\begin{array}{l}\text { Intraoperative dexmedetomidine does not reduce } \\
\text { postoperative opioid consumption or improve pain scores } \\
\text { after multilevel deformity correction spine surgery. }\end{array}$ & Naik et al., 2016 \\
\hline $\begin{array}{l}\text { Gasserian ganglion block for } \\
\text { trigeminal neuralgia }\end{array}$ & $\begin{array}{l}\text { A case of successful procedural } \\
\text { sedation using dexmedetomidine, } \\
0.8 \mu \mathrm{g} \times \mathrm{kg}^{-1} \times \mathrm{h}^{-1}\end{array}$ & $\begin{array}{l}\text { Dexmedetomidine sedation for interventional pain } \\
\text { management during procedures such as Gasserian } \\
\text { ganglion block may be useful. }\end{array}$ & Kido et al., 2014 \\
\hline
\end{tabular}

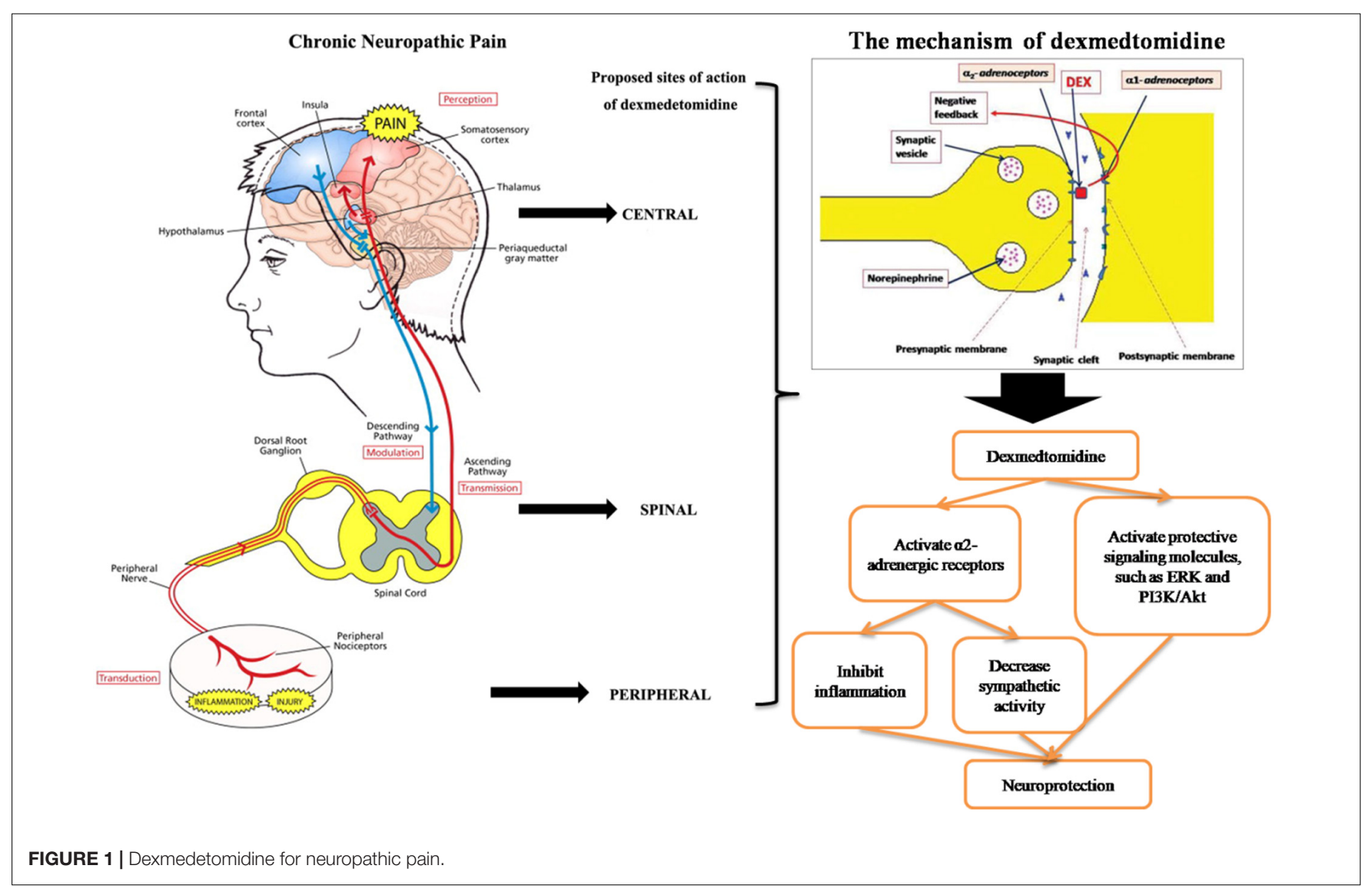

dexmedetomidine in operation. Thirteen carcinoma patients received infusion of dexmedetomidine $(0.5 \mu \mathrm{g} / \mathrm{kg} / \mathrm{h})$ and stopped infusion at the end of surgery. It was found that dexmedetomidine would significantly increase piritramide consumption than lidocaine. Dexmedetomidine failed to improve bowel function but effectively reduce propofol consumption during surgery (Andjelkovic et al., 2018). $\mathrm{Xu}$ et al. (2017) investigated whether lidocaine combined with dexmedetomidine infusion was superior in controlling pain and recovery of bowel function in 240 women undergoing elective abdominal hysterectomy. In this study, dexmedetomidine + lidocaine reduced the time to first bowel sounds, visual analog scale (VAS) score, and postoperative fentanyl requirement. The combination application significantly improved postoperative pain and enhanced recovery of bowel function (Xu et al., 2017). Naik et al. (2016) enrolled 142 patients with more than three levels of thoracic and/or lumbar spine surgery receiving dexmedetomidine of $0.5 \mu \mathrm{g} / \mathrm{kg} / \mathrm{h}$ in a randomized double-blind study. It was found that dexmedetomidine could increase the incidence of bradycardia and phenylephrine. Intraoperative dexmedetomidine does not reduce postoperative opioid consumption or improve pain 
scores after multilevel deformity correction spine surgery (Naik et al., 2016). Kido et al. (2014) reported a case of successful procedural sedation using dexmedetomidine in a 68-year-old woman undergoing left Gasserian ganglion block for intractable trigeminal neuralgia. Dexmedetomidine (DEX) was chosen to provide an effective sedation and clear communication about the injection of drugs or thermocoagulation. After $15 \mathrm{~min}$ of DEX administration at $0.8 \mu \mathrm{g} / \mathrm{kg} / \mathrm{h}$, nerve block needle insertion was bearable. The patient could feel a weakening of the sensation of her left maxilla and mandible after receiving a $0.4-\mathrm{ml}$ mepivacaine test dose. DEX sedation for interventional pain management during procedures such as Gasserian ganglion block may be useful (Kido et al., 2014). These studies provide an important theoretical basis for the application of dexmedetomidine in neuropathic pain.

\section{CONCLUSION}

Dexmedetomidine is a novel, highly selective $\alpha 2$ adrenergic receptor agonist with moderate analgesic effects (O'Neil et al., 2014). Both preoperatively and intraoperatively, dexmedetomidine can effectively alleviate the stress response caused by anesthesia, can maintain stable circulation, and can reduce the amount of anesthetic drugs (Greenberg et al., 2017). Dexmedetomidine has a good analgesic effect in animal models of acute and chronic inflammatory pain and postoperative pain and even in chronic pain patients for whom opioid analgesics are ineffective (Mantz et al., 2011). Although most studies have shown that dexmedetomidine is safe, dexmedetomidine may also

\section{REFERENCES}

Abd-Elshafy, S. K., Abdallal, F., Kamel, E. Z., Edwar, H., Allah, E. A., Maghraby, H. H. M., et al. (2019). Paravertebral dexmedetomidine in video-assisted thoracic surgeries for acute and chronic pain prevention. Pain Phys. 22, 271-280.

Alam, A., Suen, K. C., Hana, Z., Sanders, R. D., Maze, M., and Ma, D. (2017). Neuroprotection and neurotoxicity in the developing brain: an update on the effects of dexmedetomidine and xenon. Neurotoxicol. Teratol. 60, 102-116. doi: 10.1016/j.ntt.2017.01.001

Andjelkovic, L., Novak-Jankovic, V., Pozar-Lukanovic, N., Bosnic, Z., and Spindler-Vesel, A. (2018). Influence of dexmedetomidine and lidocaine on perioperative opioid consumption in laparoscopic intestine resection: a randomized controlled clinical trial. J. Int. Med. Res. 46, 5143-5154. doi: 10. 1177/0300060518792456

Barends, C. R., Absalom, A., van Minnen, B., Vissink, A., and Visser, A. (2017). Dexmedetomidine versus midazolam in procedural sedation. a systematic review of efficacy and safety. PLoS One 12:e0169525. doi: 10.1371/journal.pone. 0169525

Baron, R. (2009). Neuropathic pain: a clinical perspective. Handb. Exp. Pharmacol. 194, 3-30. doi: 10.1007/978-3-540-79090-7_1

Bell, M. T., Puskas, F., Bennett, D. T., Herson, P. S., Quillinan, N., Fullerton, D. A., et al. (2014). Dexmedetomidine, an alpha-2a adrenergic agonist, promotes ischemic tolerance in a murine model of spinal cord ischemia-reperfusion. J. Thorac. Cardiovasc. Surg. 147, 500-506.

Bettini, L., and Moore, K. (2016). Central sensitization in functional chronic pain syndromes: overview and clinical application. Pain Manag. Nurs. 17, 333-338. doi: 10.1016/j.pmn.2016.05.008

Binder, A., and Baron, R. (2016). The pharmacological therapy of chronic neuropathic pain. Dtsch Arztebl. Int. 113, 616-625. have some complications, such as bradycardia (Zhang and Bai, 2014). Therefore, strict monitoring is required in clinical use. In this review, we summarized the neuroprotective mechanisms of dexmedetomidine (Figure 1), but studies on these mechanisms are mostly limited to animal experiments (Wang et al., 2019). Therefore, in future clinical research, it is necessary to consider the influence of various drug factors on various aspects of neuronal injury and to conduct a large number of preclinical experiments to accurately assess the safety and effectiveness of dexmedetomidine on the neuroprotective effects of the human body (Wang L. et al., 2017; Wang et al., 2019). Moreover, in lieu of the neuroprotective effect of dexmedetomidine, the drug has begun to be studied in clinical neuropathic pain diseases (Kim et al., 2017; Shi et al., 2018). Thus, we have also summarized many studies on the application of dexmedetomidine in neuropathic pain in this review.

\section{AUTHOR CONTRIBUTIONS}

YZ and SW designed the concept and drafted the manuscript. JH, $\mathrm{NY}$, and CJ collected the literature, analyzed the data and edited the language in the manuscript.

\section{FUNDING}

This study is supported by the Youth Research Fund from The Affiliated Hospital of Qingdao University (for YZ) and National Natural Science Foundation of China under Grant No. 81771415.

Brouwer, B. A., Merkies, I. S., Gerrits, M. M., Waxman, S. G., Hoeijmakers, J. G., and Faber, C. G. (2014). Painful neuropathies: the emerging role of sodium channelopathies. J. Peripher. Nerv. Syst. 19, 53-65. doi: 10.1111/jns5. 12071

Chandrasekar, B., Mummidi, S., Claycomb, W. C., Mestril, R., and Nemer, M. (2005). Interleukin-18 is a pro-hypertrophic cytokine that acts through a phosphatidylinositol 3-kinase-phosphoinositide-dependent kinase-1-AktGATA4 signaling pathway in cardiomyocytes. J. Biol. Chem. 280, 4553-4567. doi: 10.1074/jbc.m411787200

Chen, J., Li, H., Lim, G., McCabe, M. F., Zhao, W., Yang, Y., et al. (2018). Different effects of dexmedetomidine and midazolam on the expression of NR2B and GABAA-alphal following peripheral nerve injury in rats. IUBMB Life 70, 143-152. doi: 10.1002/iub.1713

Cheng, J., Zhu, P., Qin, H., Li, X., Yu, H., and Peng, X. (2018). Dexmedetomidine attenuates cerebral ischemia/reperfusion injury in neonatal rats by inhibiting TLR4 signaling. J. Int. Med. Res. 46, 2925-2932. doi: 10.1177/0300060518781382

Chiu, K. M., Lin, T. Y., Lu, C. W., and Wang, S. J. (2011). Inhibitory effect of glutamate release from rat cerebrocortical nerve terminals by alpha2 adrenoceptor agonist dexmedetomidine. Eur. J. Pharmacol. 670, 137-147. doi: 10.1016/j.ejphar.2011.08.012

Cohen, S. P., and Mao, J. (2014). Neuropathic pain: mechanisms and their clinical implications. BMJ 348:f7656. doi: 10.1136/bmj.f7656

Colloca, L., Ludman, T., Bouhassira, D., Baron, R., Dickenson, A. H., Yarnitsky, D., et al. (2017). Neuropathic pain. Nat. Rev. Dis. Primers 3:17002.

Cruickshank, M., Henderson, L., MacLennan, G., Fraser, C., Campbell, M., Blackwood, B., et al. (2016). Alpha-2 agonists for sedation of mechanically ventilated adults in intensive care units: a systematic review. Health Technol. Assess. 20, 1-17. 
Crupi, R., Impellizzeri, D., and Cuzzocrea, S. (2019). Role of metabotropic glutamate receptors in neurological disorders. Front. Mol. Neurosci. 12:20. doi: 10.3389/fnmol.2019.00020

Di Cesare Mannelli, L., Micheli, L., Crocetti, L., Giovannoni, M. P., Vergelli, C., and Ghelardini, C. (2017). Alpha2 adrenoceptor: a target for neuropathic pain treatment. Mini. Rev. Med. Chem. 17, 95-107. doi: 10.2174/ 1389557516666160609065535

Dillon, R. C., Palma, J. A., Spalink, C. L., Altshuler, D., Norcliffe-Kaufmann, L., Fridman, D., et al. (2017). Dexmedetomidine for refractory adrenergic crisis in familial dysautonomia. Clin. Auton. Res. 27, 7-15. doi: 10.1007/s10286-0160383-5

Do, S. H., Park, S. J., Shin, H. J., Paik, H. S., Zuo, Z., Yoon, H. J., et al. (2014). Dexmedetomidine increases the activity of excitatory amino acid transporter type 3 expressed in Xenopus oocytes: the involvement of protein kinase $\mathrm{C}$ and phosphatidylinositol 3-kinase. Eur. J. Pharmacol. 738, 8-13. doi: 10.1016/j. ejphar.2014.05.021

Dumitrescu, L., Popescu-Olaru, I., Cozma, L., Tulba, D., Hinescu, M. E., Ceafalan, L. C., et al. (2018). Oxidative stress and the microbiota-gut-brain axis. Oxid. Med. Cell Longev. 2018:2406594.

Endesfelder, S., Makki, H., von Haefen, C., Spies, C. D., Buhrer, C., and Sifringer, M. (2017). Neuroprotective effects of dexmedetomidine against hyperoxia-induced injury in the developing rat brain. PLoS One 12:e0171498. doi: 10.1371/journal. pone. 0171498

Farghaly, H. S., Abd-Ellatief, R. B., Moftah, M. Z., Mostafa, M. G., Khedr, E. M., and Kotb, H. I. (2014). The effects of dexmedetomidine alone and in combination with tramadol or amitriptyline in a neuropathic pain model. Pain Phys. 17, 187-195.

Farghaly, H. S., Mahmoud, A. M., and Abdel-Sater, K. A. (2016). Effect of dexmedetomidine and cold stress in a rat model of neuropathic pain: Role of interleukin-6 and tumor necrosis factor-alpha. Eur. J. Pharmacol. 776, 139-145. doi: 10.1016/j.ejphar.2016.02.046

Freeman, K. A., Fullerton, D. A., Foley, L. S., Bell, M. T., Cleveland, J. C. Jr., Weyant, M. J., et al. (2015). Spinal cord protection via alpha-2 agonist-mediated increase in glial cell-line-derived neurotrophic factor. J. Thorac. Cardiovasc. Surg. 149, 578-584.

Fu, C., Dai, X., Yang, Y., Lin, M., Cai, Y., and Cai, S. (2017). Dexmedetomidine attenuates lipopolysaccharide-induced acute lung injury by inhibiting oxidative stress, mitochondrial dysfunction and apoptosis in rats. Mol. Med. Rep. 15, 131-138. doi: $10.3892 / \mathrm{mmr} .2016 .6012$

Giovannitti, J. A. Jr., Thoms, S. M., and Crawford, J. J. (2015). Alpha-2 adrenergic receptor agonists: a review of current clinical applications. Anesth. Prog. 62, 31-39.

Greenberg, R. G., Wu, H., Laughon, M., Capparelli, E., Rowe, S., Zimmerman, K. O., et al. (2017). Population pharmacokinetics of dexmedetomidine in infants. J. Clin. Pharmacol. 57, 1174-1182.

Gupta, A., and Bah, M. (2016). NSAIDs in the treatment of postoperative pain. Curr. Pain Headache Rep. 20:62.

Hoffman, W. E., Baughman, V. L., and Albrecht, R. F. (1993). Interaction of catecholamines and nitrous oxide ventilation during incomplete brain ischemia in rats. Anesth. Analg. 77, 908-912.

Hoy, S. M., and Keating, G. M. (2011). Dexmedetomidine: a review of its use for sedation in mechanically ventilated patients in an intensive care setting and for procedural sedation. Drugs 71, 1481-1501. doi: 10.2165/11207190-00000000000000

Hsieh, C. H., Lin, Y. J., Chen, W. L., Huang, Y. C., Chang, C. W., Cheng, F. C., et al. (2017). HIF-1alpha triggers long-lasting glutamate excitotoxicity via system xc() in cerebral ischaemia-reperfusion. J. Pathol. 241, 337-349. doi: 10.1002/path. 4838

Huang, X., Deng, R., Tu, W., and Hu, Z. (2017). Dexmedetomidine reduces neuropathic pain in a rat model of skin/muscle incision and retraction. Asian J. Surg. 40, 35-40. doi: 10.1016/j.asjsur.2015.10.009

Jassam, Y. N., Izzy, S., Whalen, M., McGavern, D. B., and El Khoury, J. (2017). Neuroimmunology of traumatic brain injury: time for a paradigm shift. Neuron 95, 1246-1265. doi: 10.1016/j.neuron.2017.07.010

Jiang, L., Hu, M., Lu, Y., Cao, Y., Chang, Y., and Dai, Z. (2017). The protective effects of dexmedetomidine on ischemic brain injury: a meta-analysis. J. Clin. Anesth. 40, 25-32. doi: 10.1016/j.jclinane.2017. 04.003
Kato, R. I., Tachibana, K., Hashimoto, T., Takita, K., and Morimoto, Y. (2014). Dexmedetomidine suppresses long-term potentiation in the hippocampal CA1 field of anesthetized rats. J. Anesth. 28, 828-832. doi: 10.1007/s00540-0141853-3

Keating, G. M. (2015). Dexmedetomidine: a review of its use for sedation in the intensive care setting. Drugs 75, 1119-1130. doi: 10.1007/s40265-015-0419-5

Kenney, M. J., Larsen, B. T., McMurphy, R. M., Mason, D., and Fels, R. J. (2014). Dexmedetomidine and regulation of splenic sympathetic nerve discharge. Auton Neurosci. 183, 111-115. doi: 10.1016/j.autneu.2014.02.009

Kido, H., Komasawa, N., Fujiwara, S., Hyoda, A., Morimoto, K., and Minami, T. (2014). [Gasserian ganglion block for trigeminal neuralgia under dexmedetomidine sedation]. Masui 63, 901-903.

Kim, E., Kim, H. C., Lee, S., Ryu, H. G., Park, Y. H., Kim, J. H., et al. (2017). Dexmedetomidine confers neuroprotection against transient global cerebral ischemia/reperfusion injury in rats by inhibiting inflammation through inactivation of the TLR-4/NF-kappaB pathway. Neurosci. Lett. 649, 20-27. doi: 10.1016/j.neulet.2017.04.011

Kimura, M., Saito, S., and Obata, H. (2012). Dexmedetomidine decreases hyperalgesia in neuropathic pain by increasing acetylcholine in the spinal cord. Neurosci. Lett. 529, 70-74. doi: 10.1016/j.neulet.2012.08.008

Knezevic, N. N., Tverdohleb, T., Nikibin, F., Knezevic, I., and Candido, K. D. (2017). Management of chronic neuropathic pain with single and compounded topical analgesics. Pain Manag. 7, 537-558. doi: 10.2217/pmt-2017-0020

Kuang, Y., Zhang, R. R., Pei, Q., Tan, H. Y., Guo, C. X., Huang, J., et al. (2015). Pharmacokinetic and pharmacodynamic study of dexmedetomidine in elderly patients during spinal anesthesia. Int. J. Clin. Pharmacol. Ther. 53, 1005-1014.

Lee, H. G., Choi, J. I., Kim, Y. O., and Yoon, M. H. (2013). The role of alpha-2 adrenoceptor subtype in the antiallodynic effect of intraplantar dexmedetomidine in a rat spinal nerve ligation model. Neurosci. Lett. 557(Pt B), 118-122. doi: 10.1016/j.neulet.2013.10.002

Lee, M. (2013). Neurotransmitters and microglial-mediated neuroinflammation. Curr. Protein Pept. Sci. 14, 21-32. doi: 10.2174/1389203711314010005

Lewis, S. R., Nicholson, A., Smith, A. F., and Alderson, P. (2015). Alpha2 adrenergic agonists for the prevention of shivering following general anaesthesia. Cochrane Database Syst. Rev. 2015:CD011107.

Li, C., Mo, Z., Lei, J., Li, H., Fu, R., Huang, Y., et al. (2018). Edaravone attenuates neuronal apoptosis in hypoxic-ischemic brain damage rat model via suppression of TRAIL signaling pathway. Int. J. Biochem. Cell Biol. 99, 169-177. doi: 10.1016/j.biocel.2018.03.020

Li, J., Wang, H., Dong, B., Ma, J., and Wu, X. (2017). Adding dexmedetomidine to ropivacaine for femoral nerve block inhibits local inflammatory response. Minerva Anestesiol. 83, 590-597.

Li, X., and Zhang, W. (2018). Influence of intrathecal injection with dexmedetomidine on the behavioral ability and analgesic effects on rats with neuropathic pain and expression of protein kinase $\mathrm{C}$ in the spinal dorsal horn. Exp. Ther. Med. 16, 3835-3840.

Li, Z. C., Jia, Y. P., Wang, Y., Qi, J. L., and Han, X. P. (2018). Effects of dexmedetomidine posttreatment on BDNF and VEGF expression following cerebral ischemia/reperfusion injury in rats. Mol. Med. Rep. 17, 6033-6037.

Liang, F., Liu, M., Fu, X., Zhou, X., Chen, P., and Han, F. (2017). Dexmedetomidine attenuates neuropathic pain in chronic constriction injury by suppressing NR2B, NF-kappaB, and iNOS activation. Saudi Pharm. J. 25, 649-654. doi: 10.1016/j.jsps.2017.04.039

Liao, Z., Cao, D., Han, X., Liu, C., Peng, J., Zuo, Z., et al. (2014). Both JNK and P38 MAPK pathways participate in the protection by dexmedetomidine against isoflurane-induced neuroapoptosis in the hippocampus of neonatal rats. Brain Res. Bull. 107, 69-78. doi: 10.1016/j.brainresbull.2014.07.001

Lin, J. P., Chen, C. Q., Huang, L. E., Li, N. N., Yang, Y., Zhu, S. M., et al. (2018). Dexmedetomidine attenuates neuropathic pain by inhibiting P2X7R expression and ERK phosphorylation in rats. Exp. Neurobiol. 27, 267-276. doi: 10.5607/en. 2018.27.4.267

Liu, Y. J., Wang, D. Y., Yang, Y. J., and Lei, W. F. (2017). Effects and mechanism of dexmedetomidine on neuronal cell injury induced by hypoxia-ischemia. $B M C$ Anesthesiol. 17:117. doi: 10.1186/s12871-015-0042-117

Ma, J., Chen, Q., Li, J., Zhao, H., Mi, E., Chen, Y., et al. (2018). Dexmedetomidinemediated prevention of renal ischemia-reperfusion injury depends in part on cholinergic anti-inflammatory mechanisms. Anesth. Analg. 130, 1054-1062. doi: 10.1213 /ane. 0000000000003820 
MacDonald, J. F., Jackson, M. F., and Beazely, M. A. (2006). Hippocampal longterm synaptic plasticity and signal amplification of NMDA receptors. Crit. Rev. Neurobiol. 18, 71-84. doi: 10.1615/critrevneurobiol.v18.i1-2.80

Machado, V., Haas, S. J., von Bohlen, M., Halbach, O., Wree, A., Krieglstein, K., et al. (2016). Growth/differentiation factor-15 deficiency compromises dopaminergic neuron survival and microglial response in the 6hydroxydopamine mouse model of Parkinson's disease. Neurobiol. Dis. 88, 1-15. doi: 10.1016/j.nbd.2015.12.016

Magalhaes, R. C., Moreira, J. M., Lauar, A. O., da Silva, A. A. S., Teixeira, A. L., and Acs, E. S. (2019). Inflammatory biomarkers in children with cerebral palsy: a systematic review. Res. Dev. Disabil. 95:103508. doi: 10.1016/j.ridd.2019.103508

Mahmoud, M., and Mason, K. P. (2015). Dexmedetomidine: review, update, and future considerations of paediatric perioperative and periprocedural applications and limitations. Br. J. Anaesth. 115, 171-182. doi: 10.1093/bja/ aev226

Mantz, J., Josserand, J., and Hamada, S. (2011). Dexmedetomidine: new insights. Eur. J. Anaesthesiol. 28, 3-6. doi: 10.1097/eja.0b013e32833e266d

Mavropoulos, G., Minguet, G., and Brichant, J. F. (2014). Alpha-2 adrenoreceptor agonists in anaesthesia and intensive care medicine. Rev. Med. Liege 69, 97-101.

McAdams, R. M., McPherson, R. J., Kapur, R., Phillips, B., Shen, D. D., and Juul, S. E. (2015). Dexmedetomidine reduces cranial temperature in hypothermic neonatal rats. Pediatr. Res. 77, 772-778. doi: 10.1038/pr.2015.45

Naik, B. I., Nemergut, E. C., Kazemi, A., Fernandez, L., Cederholm, S. K., McMurry, T. L., et al. (2016). The effect of dexmedetomidine on postoperative opioid consumption and pain after major spine surgery. Anesth. Analg. 122, 16461653. doi: 10.1213/ane.0000000000001226

Niittykoski, M., Haapalinna, A., and Sirvio, J. (2000). Diminution of N-methyl$\mathrm{D}$-aspartate-induced perturbation of neurotransmission by dexmedetomidine in the CA1 field of rat hippocampus in vitro. Neurosci. Lett. 281, 95-98. doi: 10.1016/s0304-3940(00)00811-9

Obata, H. (2017). Analgesic mechanisms of antidepressants for neuropathic pain. Int. J. Mol. Sci. 18, 1-10.

O’Neil, T., Rodgers, P. E., and Shultz, C. (2014). Dexmedetomidine as adjuvant therapy for acute postoperative neuropathic pain crisis. J. Palliat. Med. 17, 1164-1166. doi: 10.1089/jpm.2013.0556

Park, H. J., Kim, Y. H., Koh, H. J., Park, C. S., Kang, S. H., Choi, J. H., et al. (2012). Analgesic effects of dexmedetomidine in vincristine-evoked painful neuropathic rats. J. Korean Med. Sci. 27, 1411-1417.

Pichot, C., Longrois, D., Ghignone, M., and Quintin, L. (2012). Dexmedetomidine and clonidine: a review of their pharmacodynamy to define their role for sedation in intensive care patients. Ann. Fr. Anesth. Reanim. 31, 876-896.

Pocock, J. M., and Kettenmann, H. (2007). Neurotransmitter receptors on microglia. Trends Neurosci. 30, 527-535. doi: 10.1016/j.tins.2007.07.007

Qian, Y., Wang, Q., Jiao, J., Wang, G., Gu, Z., Huang, D., et al. (2019). Intrathecal injection of dexmedetomidine ameliorates chronic neuropathic pain via the modulation of MPK3/ERK1/2 in a mouse model of chronic neuropathic pain. Neurol. Res. 41, 1059-1068. doi: 10.1080/01616412.2019.1672391

Rodriguez-Gonzalez, R., Sobrino, T., Veiga, S., Lopez, P., Rodriguez-Garcia, J., del Rio, S. V., et al. (2016). Neuroprotective effects of dexmedetomidine conditioning strategies: Evidences from an in vitro model of cerebral ischemia. Life Sci. 144, 162-169. doi: 10.1016/j.lfs.2015.12.007

Salim, S. (2017). Oxidative stress and the central nervous system. J. Pharmacol. Exp. Ther. 360, 201-205.

Sato, K., Kimura, T., Nishikawa, T., Tobe, Y., and Masaki, Y. (2010). Neuroprotective effects of a combination of dexmedetomidine and hypothermia after incomplete cerebral ischemia in rats. Acta Anaesthesiol. Scand. 54, 377-382. doi: 10.1111/j.1399-6576.2009.02139.x

Scholz, J., Finnerup, N. B., Attal, N., Aziz, Q., Baron, R., Bennett, M. I., et al. (2019). The IASP classification of chronic pain for ICD-11: chronic neuropathic pain. Pain 160, 53-59.

Shen, M., Wang, S., Wen, X., Han, X. R., Wang, Y. J., Zhou, X. M., et al. (2017). Dexmedetomidine exerts neuroprotective effect via the activation of the $\mathrm{PI} 3 \mathrm{~K} / \mathrm{Akt} / \mathrm{mTOR}$ signaling pathway in rats with traumatic brain injury. Biomed. Pharmacother. 95, 885-893. doi: 10.1016/j.biopha.2017.08.125

Shi, Y., Peng, X. H., Li, X., Luo, G. P., and Wu, M. F. (2018). Neuroprotective role of dexmedetomidine pretreatment in cerebral ischemia injury via ADRA2Amediated phosphorylation of ERK1/2 in adult rats. Exp. Ther. Med. 16, 52015209.
Singh, A., and Abraham, W. C. (2017). Astrocytes and synaptic plasticity in health and disease. Exp. Brain Res. 235, 1645-1655. doi: 10.1007/s00221-0 17-4928-1

Sottas, C. E., and Anderson, B. J. (2017). Dexmedetomidine: the new all-in-one drug in paediatric anaesthesia? Curr. Opin. Anaesthesiol. 30, 441-451. doi: 10.1097/aco.0000000000000488

Stacey, B. R. (2005). Management of peripheral neuropathic pain. Am. J. Phys. Med. Rehabil. 84(3 Suppl.), S4-S16.

Starke, K. (2014). History of catecholamine research. Chem. Immunol. Allergy 100, 288-301. doi: 10.1159/000359962

Sun, K., Fan, J., and Han, J. (2015). Ameliorating effects of traditional Chinese medicine preparation, Chinese materia medica and active compounds on ischemia/reperfusion-induced cerebral microcirculatory disturbances and neuron damage. Acta Pharm. Sin B 5, 8-24. doi: 10.1016/j.apsb.2014.11.002

Tachibana, K., Hashimoto, T., Kato, R., Uchida, Y., Ito, R., Takita, K., et al. (2012). Neonatal administration with dexmedetomidine does not impair the rat hippocampal synaptic plasticity later in adulthood. Paediatr. Anaesth. 22, 713-719. doi: 10.1111/j.1460-9592.2012.03810.x

Takamatsu, I., Iwase, A., Ozaki, M., Kazama, T., Wada, K., and Sekiguchi, M. (2008). Dexmedetomidine reduces long-term potentiation in mouse hippocampus. Anesthesiology 108, 94-102. doi: 10.1097/01.anes.0000296076. 04510.e1

Torres-Cuevas, I., Corral-Debrinski, M., and Gressens, P. (2019). Brain oxidative damage in murine models of neonatal hypoxia/ischemia and reoxygenation. Free Radic. Biol. Med. 142, 3-15. doi: 10.1016/j.freeradbiomed.2019. 06.011

Ulger, F., Bozkurt, A., Bilge, S. S., Ilkaya, F., Dilek, A., Bostanci, M. O., et al. (2009). The antinociceptive effects of intravenous dexmedetomidine in colorectal distension-induced visceral pain in rats: the role of opioid receptors. Anesth. Analg. 109, 616-622. doi: 10.1213/ane.0b013e3181a9fae2

von Bernhardi, R., Eugenin-von Bernhardi, J., Flores, B., and Eugenin Leon, J. (2016). Glial cells and Integrity of the nervous system. Adv. Exp. Med. Biol. 949, 1-24. doi: 10.1007/978-3-319-40764-7_1

Vranken, J. H. (2012). Elucidation of pathophysiology and treatment of neuropathic pain. Cent. Nerv. Syst. Agents Med. Chem. 12, 304-314. doi: 10. 2174/187152412803760645

Wang, L., Liu, H., Zhang, L., Wang, G., Zhang, M., and Yu, Y. (2017). Neuroprotection of dexmedetomidine against cerebral ischemia-reperfusion injury in rats: involved in inhibition of NF-kappaB and inflammation response. Biomol. Ther. 25, 383-389. doi: 10.4062/biomolther.2015.180

Wang, Q., Yang, Q., and Wu, W. (2020). Graphene-based steganographic aptasensor for information computing and monitoring toxins of biofilm in food. Front. Micobiol. 10:3139. doi: 10.3389/fmicb.2019.03139

Wang, S. L., Duan, L., Xia, B., Liu, Z., Wang, Y., and Wang, G. M. (2017). Dexmedetomidine preconditioning plays a neuroprotective role and suppresses TLR4/NF-kappaB pathways model of cerebral ischemia reperfusion. Biomed. Pharmacother. 93, 1337-1342. doi: 10.1016/j.biopha.2017.06.051

Wang, X., Ji, J., Fen, L., and Wang, A. (2013). Effects of dexmedetomidine on cerebral blood flow in critically ill patients with or without traumatic brain injury: a prospective controlled trial. Brain Inj. 27, 1617-1622. doi: 10.3109/ 02699052.2013.831130

Wang, X., Shan, Y., Tang, Z., Gao, L., and Liu, H. (2019). Neuroprotective effects of dexmedetomidine against isoflurane-induced neuronal injury via glutamate regulation in neonatal rats. Drug Des. Devel. Ther. 13, 153-160. doi: 10.2147/ dddt.s163197

Watzlawik, J., Warrington, A. E., and Rodriguez, M. (2010). Importance of oligodendrocyte protection, BBB breakdown and inflammation for remyelination. Expert Rev. Neurother. 10, 441-457. doi: 10.1586/ern.10.13

Weerink, M. A. S., Struys, M., Hannivoort, L. N., Barends, C. R. M., Absalom, A. R., and Colin, P. (2017). Clinical pharmacokinetics and pharmacodynamics of dexmedetomidine. Clin. Pharmacokinet. 56, 893-913.

Wu, J., Vogel, T., Gao, X., Lin, B., Kulwin, C., and Chen, J. (2018). Neuroprotective effect of dexmedetomidine in a murine model of traumatic brain injury. Sci. Rep. 8:4935.

Wu, W., Yu, C., Chen, J., and Yang, Q. (2020). Fluorometric detection of copper ions using click chemistry and the target-induced conjunction of split DNAzyme fragments. Int. J. Environ. Ann. 100, 324-332. doi: 10.1080/ 03067319.2019 .1636977 
Xu, J., Lei, S., and Ye, G. (2019). Dexmedetomidine attenuates oxidative/nitrative stress in lung tissues of septic mice partly via activating heme oxygenase-1. Exp. Ther. Med. 18, 3071-3077.

Xu, S. Q., Li, Y. H., Wang, S. B., Hu, S. H., Ju, X., and Xiao, J. B. (2017). Effects of intravenous lidocaine, dexmedetomidine and their combination on postoperative pain and bowel function recovery after abdominal hysterectomy. Minerva Anestesiol. 83, 685-694.

Yan, Y. Y., Li, C. Y., Zhou, L., Ao, L. Y., Fang, W. R., and Li, Y. M. (2017). Research progress of mechanisms and drug therapy for neuropathic pain. Life Sci. 190, 68-77. doi: 10.1016/j.lfs.2017.09.033

Yang, L., Tang, J., Dong, J., and Zheng, J. (2015). Alpha2-adrenoceptorindependent inhibition of acetylcholine receptor channel and sodium channel by dexmedetomidine in rat superior cervical ganglion neurons. Neuroscience 289, 9-18. doi: 10.1016/j.neuroscience.2014.12.045

Yang, Y., Xia, Z., Meng, Q., Liu, K., Xiao, Y., and Shi, L. (2018). Dexmedetomidine relieves neuropathic pain by inhibiting hyperpolarization-activated cyclic nucleotide-gated currents in dorsal root ganglia neurons. Neuroreport 29, 1001-1006. doi: 10.1097/wnr.0000000000001068

Zhang, F., Ma, X. L., Wang, Y. X., He, C. C., Tian, K., Wang, H. G., et al. (2017). TPEN, a Specific $\mathrm{Zn}(2+)$ chelator, inhibits sodium dithionite and glucose deprivation (SDGD)-induced neuronal death by modulating apoptosis, glutamate signaling, and voltage-gated $\mathrm{K}(+)$ and $\mathrm{Na}(+)$ channels. Cell Mol. Neurobiol. 37, 235-250. doi: 10.1007/s10571-016-0364-1

Zhang, J., Wang, Z., Wang, Y., Zhou, G., and Li, H. (2015). The effect of dexmedetomidine on inflammatory response of septic rats. BMC Anesthesiol. 15:68. doi: 10.1186/s12871-015-0042-8

Zhang, X., and Bai, X. (2014). New therapeutic uses for an alpha2 adrenergic receptor agonist-dexmedetomidine in pain management. Neurosci. Lett. 561, 7-12. doi: 10.1016/j.neulet.2013.12.039

Zhenyukh, O., Civantos, E., Ruiz-Ortega, M., Sanchez, M. S., Vazquez, C., Peiro, C., et al. (2017). High concentration of branched-chain amino acids promotes oxidative stress, inflammation and migration of human peripheral blood mononuclear cells via mTORC1 activation. Free Radic. Biol. Med. 104, 165-177. doi: 10.1016/j.freeradbiomed.2017.01.009

Zhong, J. M., Lu, Y. C., and Zhang, J. (2018). Dexmedetomidine reduces diabetic neuropathy pain in rats through the Wnt 10a/beta-catenin signaling pathway. Biomed. Res. Int. 2018:9043628.

Zhou, T. T., Wu, J. R., Chen, Z. Y., Liu, Z. X., and Miao, B. (2014). Effects of dexmedetomidine on P2X4Rs, p38-MAPK and BDNF in spinal microglia in rats with spared nerve injury. Brain Res. 1568, 21-30. doi: 10.1016/j.brainres. 2014.04.025

Zhou, W. L., Zhang, J. T., Xu, W., and Sun, J. H. (2019). Protective effects of polygalasaponin $\mathrm{F}$ on oxidative stress and apoptosis-induced ischemic myocardial injury in neonatal rats with hypoxic-ischemic brain damage. Neuroreport 30, 1148-1156. doi: 10.1097/wnr.0000000000001330

Zhu, Y. M., Wang, C. C., Chen, L., Qian, L. B., Ma, L. L., Yu, J., et al. (2013). Both PI3K/Akt and ERK1/2 pathways participate in the protection by dexmedetomidine against transient focal cerebral ischemia/reperfusion injury in rats. Brain Res. 1494, 1-8. doi: 10.1016/j.brainres.2012.11.047

Zindler, E., and Zipp, F. (2010). Neuronal injury in chronic CNS inflammation. Best Pract. Res. Clin. Anaesthesiol. 24, 551-562. doi: 10.1016/j.bpa.2010.11.001

Zuchero, J. B., and Barres, B. A. (2015). Glia in mammalian development and disease. Development 142, 3805-3809. doi: 10.1242/dev.129304

Conflict of Interest: The authors declare that the research was conducted in the absence of any commercial or financial relationships that could be construed as a potential conflict of interest.

Copyright (c) 2020 Zhao, He, Yu, Jia and Wang. This is an open-access article distributed under the terms of the Creative Commons Attribution License (CC BY). The use, distribution or reproduction in other forums is permitted, provided the original author(s) and the copyright owner(s) are credited and that the original publication in this journal is cited, in accordance with accepted academic practice. No use, distribution or reproduction is permitted which does not comply with these terms. 